\title{
Genomics and transcriptomics in veterinary oncology (Review)
}

\author{
BRIDGET MARIE HARRISON and PANAYIOTIS LOUKOPOULOS
}

\author{
Melbourne Veterinary School, Faculty of Veterinary and Agricultural Sciences, \\ University of Melbourne, Werribee, Victoria 3030, Australia
}

Received January 6, 2020; Accepted February 3, 2021

DOI: $10.3892 / \mathrm{ol} .2021 .12597$

\begin{abstract}
The sequencing of the canine genome, combined with additional genomic technologies, has created opportunities for research linking veterinary genomics with naturally occurring cancer in dogs. Also, as numerous canine cancers have features in common with human cancers, comparative studies can be performed to evaluate the use of cancers in dogs as models for human cancer. There have been several reviews of veterinary genomics but, to the best of our knowledge, there has been no comprehensive review of the literature of canine cancer genomics. PubMed and CAB Abstracts databases were searched to retrieve relevant literature using the search terms 'veterinary', 'cancer' or 'oncology', and 'genomics' or 'transcriptomics'. Results were manually assessed and grouped based on the techniques used, the cancer type investigated and genomic lesions targeted. The search resulted in the retrieval of 44 genomic and transcriptomic studies, with the most common technique employed being comparative genomic hybridization. Across both fields, the most commonly studied cancer type was canine osteosarcoma. Genomic and transcriptomic aberrations in canine cancer often reflected those reported in the corresponding human cancers. Analysis of the literature indicated that employing genomic and transcriptomic technologies has been instrumental in developing the understanding of the origin, development and pathogenesis of several canine cancers. However, their use in canine oncology is at an early phase, and there appears to be comparatively little understanding of certain canine cancer types in contrast to their human forms. Aberrations detected in all tumors were tabulated, and the results for osteosarcoma, lymphoma and leukemia, mast cell tumor, transmissible venereal tumor and urothelial carcinoma discussed in detail.
\end{abstract}

Correspondence to: Dr Panayiotis Loukopoulos, Melbourne Veterinary School, Faculty of Veterinary and Agricultural Sciences, University of Melbourne, 250 Princes Highway, Werribee, Victoria 3030, Australia

E-mail: panos.loukopoulos@unimelb.edu.au

Key words: cancer, canine, genomics, oncology, One Health, transcriptomics

\section{Contents}

1. Introduction

2. Materials and methods

3. Genomics

4. Transcriptomics

5. Studies pertaining to the most common cancer types

6. Review articles

7. Conclusions

\section{Introduction}

Cancer is a leading cause of death of dogs, representing $27 \%$ of all deaths in pure bred dogs in the UK in one mortality study and approximately half of all deaths in $\operatorname{dog} s>10$ years of age (1). The advantages of using canine cancer as a model for human cancer are multifaceted (2) and offer a prime example of the benefits of One Health approaches in the study of both human and animal disease. Tumors in dogs have a similar incidence to those in humans and do not require exogenous factors as they often do in mice (2). Not only do canine tumors occur naturally and under similar environmental conditions to those in humans, a number of types of tumor also have similar histopathological and biological behaviors $(2,3)$. The clinical presentation is often similar in dogs and humans, as are a number of aspects of clinical pathology (4). Since numerous pure breeds of dog have closed populations, there is relatively high homogeneity in these animals $(2,4)$. The lack of genetic diversity results in fewer deleterious alleles being required to induce a cancer in pure breeds (4). Therefore, it is easier to map these alleles, determine which are causative and compare them with a human genome with the same cancer type. In addition, dogs also have shorter lifespans than humans, and therefore, clinical trials and cohort studies can be conducted in much shorter time periods than are required for human trials $(2,4)$.

The fields of genomics $(5,6)$ and transcriptomics have undergone rapid growth in the last two decades, particularly with the completion of the Human Genome Project in 2003. The first full canine genome was subsequently published in 2005 (7). Since then, there have been numerous technological breakthroughs in the field of genomics and transcriptomics, and hence in the ability to sequence and understand the genetic makeup of the genome, as well as the proteins it encodes. In the veterinary field, this information has been used to inform 
breeding decisions, the classification of species, disease resistance and the pathogenesis and diagnosis of diseases, including the identification of biomarkers.

A number of techniques have been employed to study the genome and transcriptome. Comparative genomic hybridization $(\mathrm{CGH})$ is one of the more commonly used techniques in veterinary genomics. In contrast to karyotyping and fluorescence in-situ hybridization (FISH), CGH does not require prior knowledge of a particular aberration to locate abnormalities, but scans the entire genome for copy number aberrations (CNAs). The test genome is compared with the control genome, and the intensity of fluorescence is indicative of the magnitude of the change in copy number. This technology has been further developed by introducing $\mathrm{CGH}$ into microarrays, which use smaller fragments of genetic material to increase the specificity of the process (8). The benefit of this technology is its ability to identify biomarkers to aid the diagnosis of multiple canine cancers by locating specific aberrations and patterns present in the cancer genome. Improved methods of diagnosis have also led to an enhanced understanding of tumorigenesis and the pathology of cancers, and in some cases, specifically targeted treatments. Canine cancer studies are not only of benefit to dogs, as some canine tumors have been shown to be acceptable models for studying similar human cancers, while results extrapolated from human cancer studies have prioritized certain research avenues in the study of canine cancer avenues, hence highlighting the very definition of One Health. This has resulted in the expansion of the field of comparative oncology.

The present review was prompted by the multiple approaches used in genomic and transcriptomic studies in veterinary medicine, which suggest that a closer examination of the commonalities between canine and human oncology is merited. The review examines the veterinary literature and establishes the technology used, the types of cancer investigated and the lesions of the genome targeted, thus identifying where gaps in the literature exist and providing an overview of further areas that need to be addressed.

\section{Materials and methods}

A literature search was completed on January 14, 2019 using the public databases CAB Abstracts ${ }^{\circledR}$ and PubMed. The search terms included 'veterinary', 'cancer' or 'oncology' and 'genomics'. The PubMed search produced 550 results, while the CAB Abstracts search produced 96 results. A second search was then completed using the terms 'veterinary', 'cancer' or 'oncology' and 'transcriptomics'. The PubMed and CAB Abstract searches produced 28 and 12 results, respectively. Both searches were conducted with an open-ended time frame. Papers that were not written in or translated into English were eliminated. The results were then manually assessed to eliminate studies that did not include veterinary species or were based on technology in other fields, such as proteomics. Studies based on animal models for the study of purely human diseases were also excluded. The remaining papers were then classified into three categories, namely genomic primary research studies, transcriptomic primary research studies and review papers.
In the first two categories the studies were then tabulated based on the type of cancer studied, techniques used, target lesion of the genome and outcome of the study. An additional search was carried out on May 13, 2020 and a further 15 suitable papers were retrieved and included in the present review.

\section{Genomics}

A summary of the 26 genomics studies retrieved, based on the cancer type studied, technique used, target lesion of the genome and outcome of the study is shown in Table I. Of these studies, seven focused on canine osteosarcoma, three on types of lymphomas and leukemias, two each on mast cell tumors (MCTs), glioma, hemangiosarcoma and canine transmissible venereal tumors (TVTs) and one each on urothelial carcinoma, histiocytic sarcoma, meningioma, prostate cancer, mammary carcinoma, pheochromocytoma and paraganglioma, ocular squamous cell carcinoma and Tasmanian devil facial tumor disease. The most commonly used technologies in these studies were $\mathrm{CGH}$ /array-based $\mathrm{CGH}(\mathrm{aCGH})$, followed by FISH, DNA microarray, whole genome sequencing, quantitative PCR (qPCR), whole exome sequencing and analysis/linear modelling.

\section{Transcriptomics}

A summary of the 13 transcriptomics studies retrieved, tabulated based on the cancer type studied, technique used, target lesion and outcome of the study is shown in Table II. In these transcriptomics studies, canine osteosarcoma was the most commonly studied type of cancer, being the subject of three of the retrieved papers, followed by nine other tumor types. The remaining studies comprised two papers on squamous cell carcinoma of the horn in cattle, one study each on canine oral melanoma, MCT, lymphoma, soft tissue sarcoma, urothelial carcinoma, mammary carcinoma and splenic hemangiosarcoma, and one study that investigated various cancer types. The techniques used included reverse transcription-PCR (RT-PCR) or RT-qPCR, microarray technology, quantitative nuclease protection assay (qNPA) technology and differential display PCR (ddPCR).

Among these studies, the most frequently used technology was $\mathrm{CGH} / \mathrm{aCGH}$, which featured in approximately half of the studies, followed by FISH and DNA microarray, whole genome sequencing, qPCR and whole exome sequencing. $\mathrm{CGH}$ is particularly useful as it enables researchers to identify copy number changes by comparing sample tumor genomes with control genomes, and hence determine which changes are consistent among tumor populations and may, therefore, be markers of disease. Microarray CGH has also been employed, enabling researchers to locate smaller aberrations (9).

All studies included in the present review were on canine tumors, with the exception of two studies on bovine squamous cell carcinoma, one on equine squamous cell carcinoma and one on Tasmanian devil facial tumor disease. Studies pertaining to the most commonly investigated tumor types are discussed below according to tumor type, in decreasing order of frequency based on the number of studies published on each tumor type. 


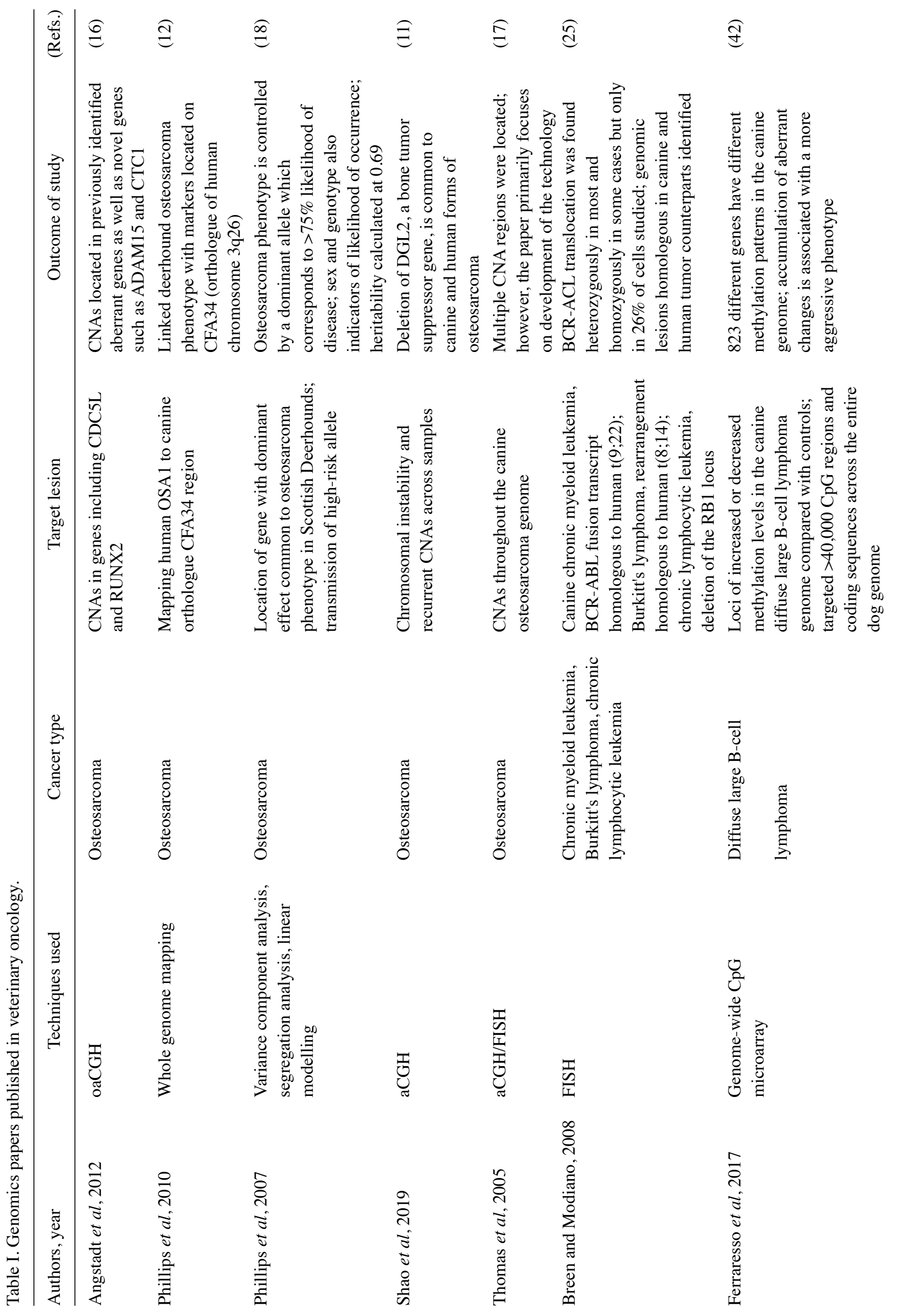




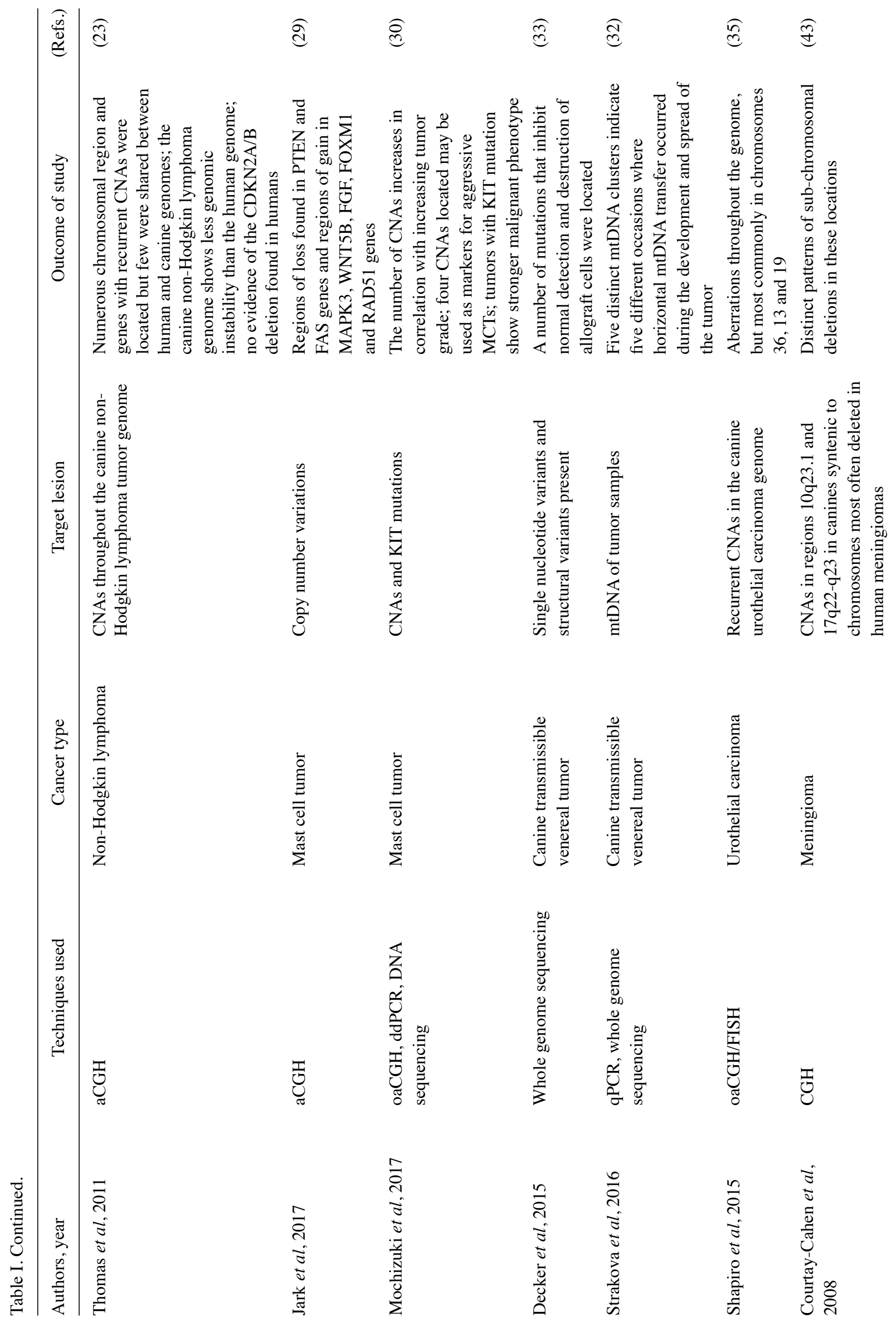




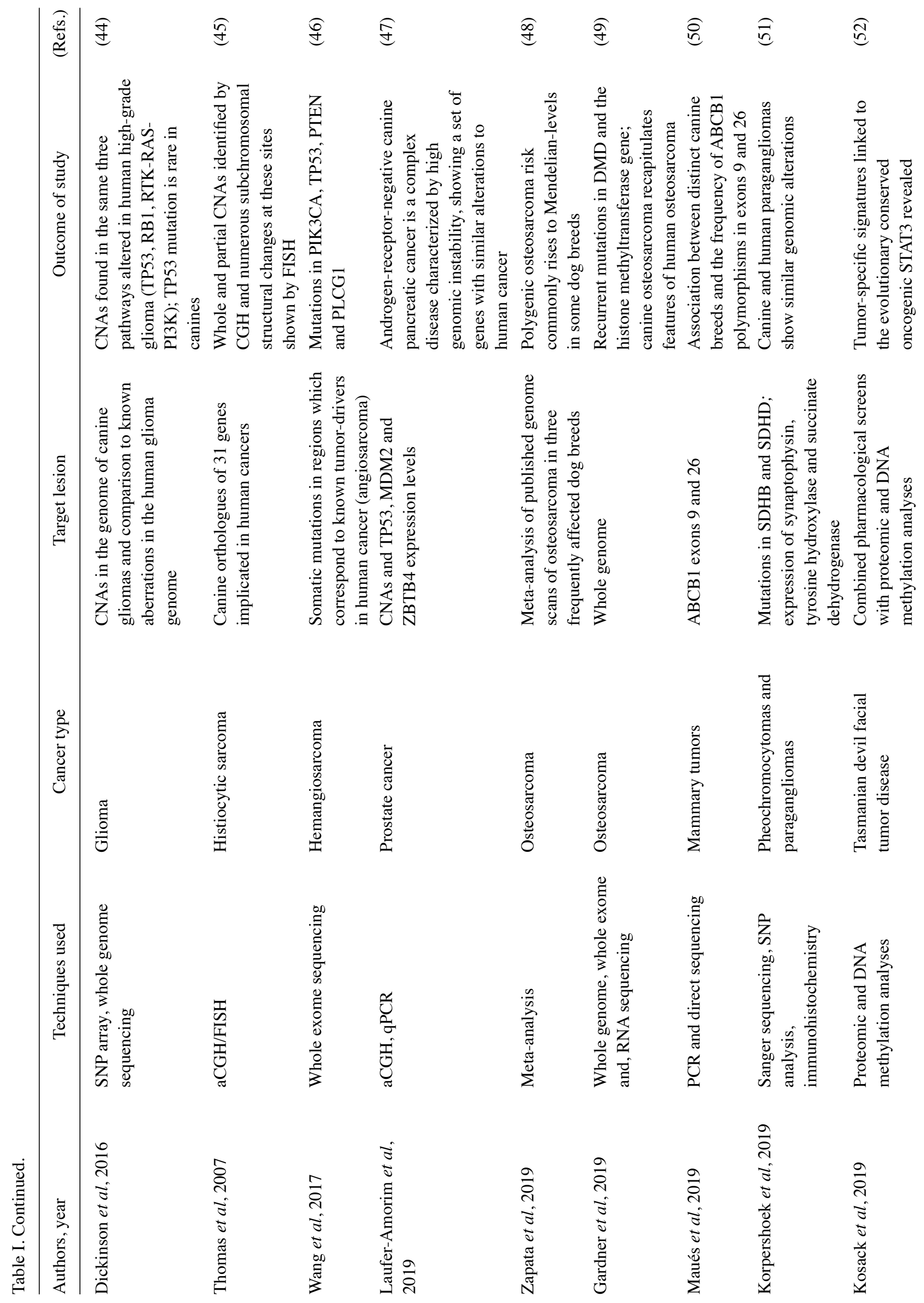




\section{Studies pertaining to the most common tumor types}

Canine osteosarcoma. Canine osteosarcoma is a common cancer in dogs. Due to the biological behavior and high prevalence of osteosarcoma in dogs, which is 27-fold higher than that in humans (10), in addition to its radiographic and histological features and patterns of metastases that are similar to those in humans (10), canine osteosarcoma has been suggested to be an appropriate animal model for human osteosarcoma. In total, there are 900 genes that have been associated with osteosarcoma $(10,11)$. In humans, most cases are not heritable and only two heritable genetic loci have been found (10), whereas in comparison, there are at least 34 heritable genetic loci in four breeds of dogs are associated with osteosarcoma (10). However, human and canine osteosarcoma have similar risk factors for development of the neoplasia. Osteosarcoma is more common in males in humans and numerous dog breeds, taller humans and large/giant dogs are predisposed $(10,12)$, and breed and race are also risk factors. Neutering status is a risk factor in dogs, with neutered animals at higher risk; although this is not applicable in humans, it suggests an association of osteosarcoma with hormonal changes in both humans and canines (10).

In canine osteosarcoma, the majority of cases occur in the appendicular skeleton, particularly the forelimb, and at the time of diagnosis, it is estimated that $80 \%$ of dogs already have metastatic disease (13). Factors that indicate a poor prognosis include high levels of serum alkaline phosphatase, systemic spread, increased tumor size, youth and high histologic grading $(13,14)$. The clinical diagnosis is usually confirmed by either a histological (14) or cytological examination (15).

In the literature reviewed, several different genetic techniques were used to investigate the genomics and transcriptomics of osteosarcoma. In one study, oligonucleotide aCGH (oaCGH) was used to determine genetic changes common to human and canine osteosarcoma (16). CNAs were located in canine osteosarcoma in the RUNX family transcription factor 2, cyclin-dependent kinase-like 5, MYC, cyclin-dependent kinase inhibitor (CDKN) $2 \mathrm{~A}$ and CDKN2B genes, previously reported to be altered in human osteosarcoma, as well as a number of additional genes (16). However, the authors noted the difficulty in differentiating genetic changes that are causative and changes that are coincidental or a result of the neoplastic process (16), a drawback shared by all similar studies. In another study (17), aCGH was also employed to locate CNAs throughout the canine cancer genome, and was combined with single locus probe (SLP) FISH analysis to give a more accurate interpretation of the presence of a CNA in a particular locus in the tumor population. This is because SLP determines the presence or absence of an aberration cell by cell, whereas aCGH only detects it in the global population and it may be averaged out to a normal level if there are both insertions and deletions in the tumor (17). aCGH was also employed to assess the deletion status of the bone tumor suppressor gene disks large homolog 2, common to both human and canine osteosarcoma, which were found to occur in 42 and $56 \%$ of cases, respectively (11). Research has also been carried out to link genes involved in osteosarcoma with their human or canine orthologues. Scottish Deerhounds are a giant breed of dogs that exhibit a high incidence of bone cancer. In a study 


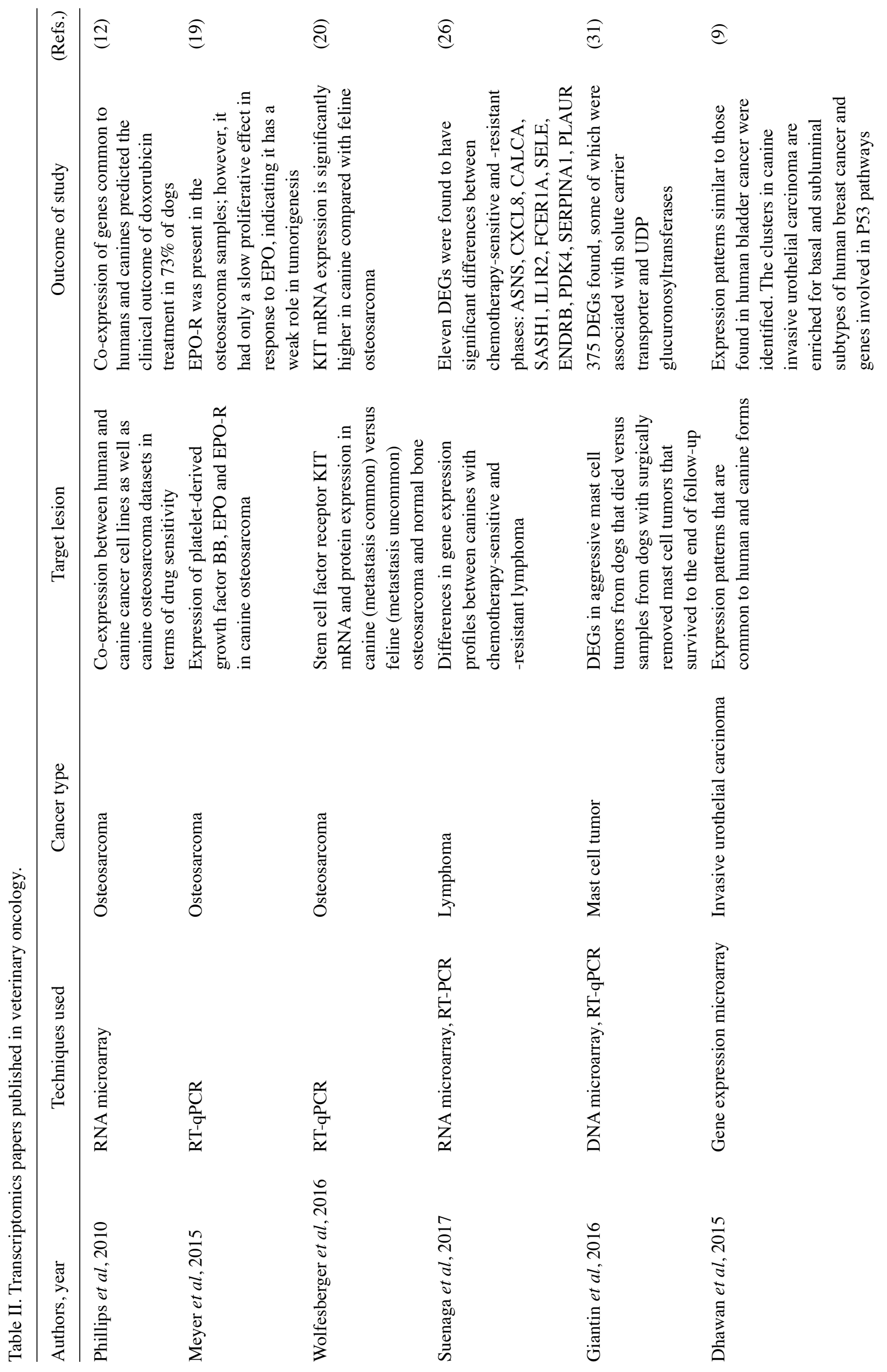




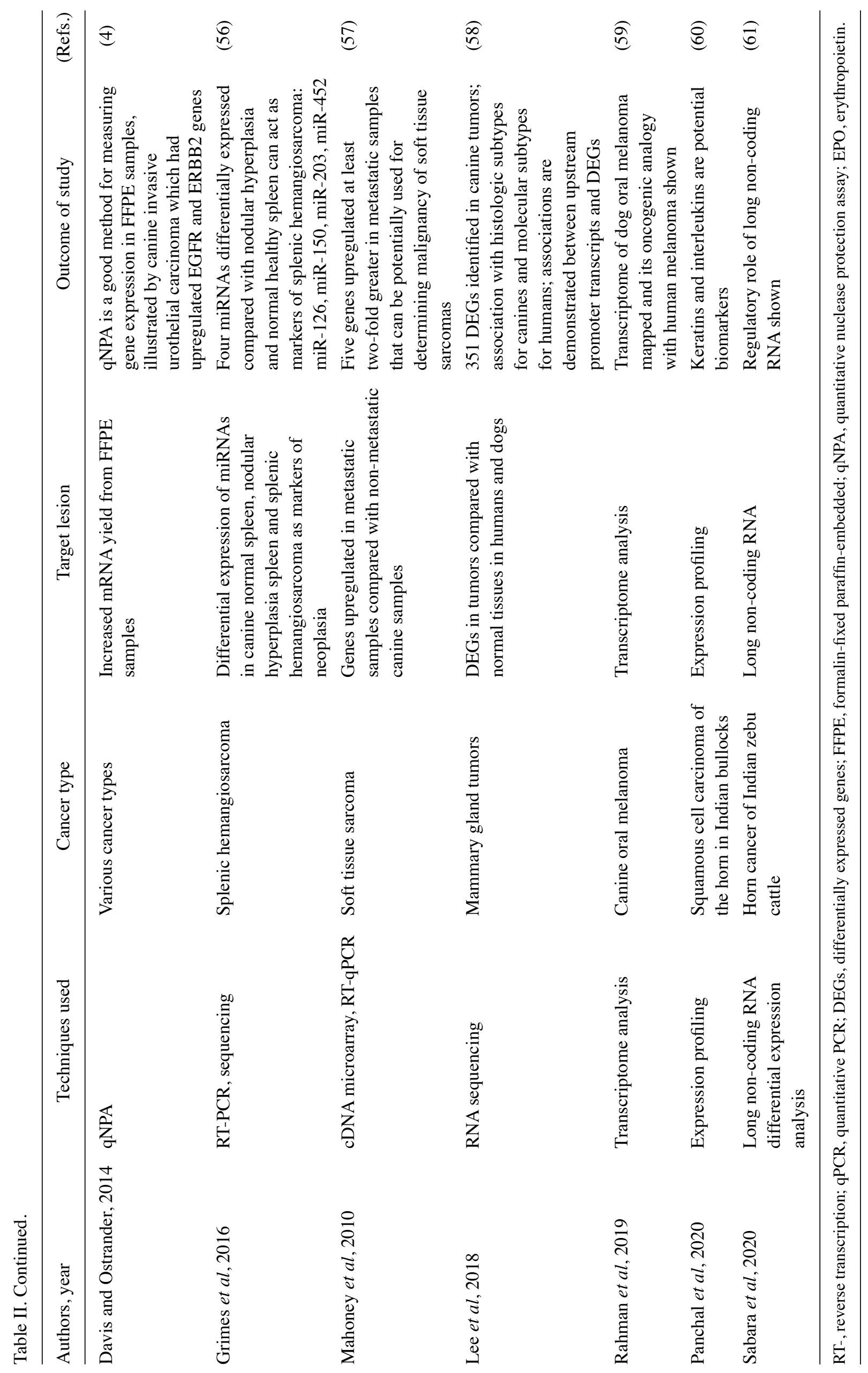


continuing earlier work (18), which suggested that osteosarcoma within this breed can be explained by the presence of a major gene of dominant effect in the Deerhound genome that contributes to the osteosarcoma phenotype, whole genome mapping was used to show that a novel locus for canine osteosarcoma maps to chromosome CFA34, the canine orthologue of human $3 \mathrm{q} 26$, in which several candidate tumor suppressor genes have been identified (12).

In the last decade, transcriptomics has also served a role in canine and human osteosarcoma research. Two studies $(19,20)$ employed RT-qPCR to determine the differential expression of genes, one of which (19) assessed erythropoietin receptor (EPO-R) gene expression in canine and feline osteosarcomas. While both dogs and cats develop osteosarcoma, the occurrence of metastasis is vastly different between the two species, with the canine form frequently exhibiting metastasis (80-90\%) while the feline form is rarely (5-10\%) metastatic (21). The EPO-R has been found to be expressed on neoplastic cells in certain types of tumors, and is therefore considered to potentially play a role in tumor progression through an autocrine mechanism. On this basis, the expression of EPO, EPO-R and platelet-derived growth factor BB was analyzed in feline and canine osteosarcomas using immunohistochemistry and RT-qPCR, to evaluate a potential association between their expression levels and metastatic potential. The study detected EPO-R expression in canine and feline tumor samples, but suggested only a minimal role for EPO-R in tumor development in both species (19). The expression of stem cell growth factor receptor KIT, quantified by RT-qPCR and immunohistochemistry, was observed to be significantly higher in canine than in feline osteosarcoma samples, indicating that it may be involved in the higher aggressiveness of canine osteosarcoma compared with feline osteosarcoma (20).

Osteosarcoma is often treated with adjuvant doxorubicin or carboplatin following amputation of the affected limb. Intraand interspecies gene-expression based models have been used to predict chemosensitivity and treatment outcome in canine osteosarcoma, with evaluation conducted using microarray gene expression and drug sensitivity data from human and canine cancer cell lines, and canine osteosarcoma tumor datasets. These models were shown to successfully predict the sensitivity of canine osteosarcoma to treatment, a finding that may facilitate improved outcomes in dogs and serve as a pre-clinical validation method in human cancer research (22).

Leukemia and lymphoma. Not all CNAs detected in canine tumor genomes are applicable or comparable to the corresponding human forms. A number of regions showing CNAs throughout the canine non-Hodgkin lymphoma (NHL) genome identified in one study exhibited very little association with aberrations in the human NHL genome, including the CDKN2A/B deletion (23). The conclusion was that the stability of the canine tumor genome in NHL was greater than that of the corresponding human form (23).

FISH is an early technology used to study chromosomal changes, such as translocations and deletions, and compare them with known changes in human cancer genomes (24). FISH was employed in one study to map changes in canine tumor genomes in canine chronic myeloid leukemia, Burkitt's lymphoma and chronic lymphocytic leukemia, and compare them with changes in the corresponding human cancers (25). In canines with Burkitt's lymphoma, a rearrangement homologous to the human $t(8 ; 14)$ one was found, and for canine chronic lymphocytic leukemia, the deletion at the RB transcriptional corepressor 1 locus known to occur in the human form was also located. However, in canines with chronic myeloid leukemia, a BCR-ABL fusion transcript shown to be homologous to the human $\mathrm{t}(9 ; 22)$ variant was only present in $26 \%$ of cells, indicating significant differences between this canine cancer and the human form (25).

RT-PCR technology has been used to study the differential gene expression profiles and differentially expressed genes (DEGs) in dogs with multicentric high-grade B-cell lymphoma (26), at the chemotherapy-sensitive and -resistant stages. A total of 57 DEGs were identified and 16 of them were associated with chemotherapy-resistance. Importantly, 11 genes with significant changes in expression levels were detected in seven dogs with chemotherapy-resistant lymphoma.

Canine MCTs. MCTs are the most common cutaneous cancers in the canine species $(27,28)$ and are present in $0.27 \%$ of the population (28). Humans are also susceptible to this type of tumor; hence, again, the naturally high prevalence in dogs makes them a good candidate for a comparative oncology approach. One study used aCGH to search for CNAs in the genomic DNA of MCTs from two populations of dogs in which the MCTs exhibited differing biological behaviors; one comprised dogs that survived $<6$ months from the date of diagnosis and the other comprised dogs that survived $>12$ months (29). Particular CNAs were found to be present significantly more frequently in the population with the shorter survival time; these included regions of gain in MAPK3, WNT5B, fibroblast growth factor, forkhead box M1 and RAD51 recombinase genes and regions of loss in PTEN and FAS genes (29).

Similar CNAs were identified in human and canine cancer MCTs in a study that used aCGH to locate CNAs in the canine cancer genome over a large sample size $(n=109)$ including multiple breed types (30), which strengthened the validity of the study. This study found a correlation between the number of CNAs present in the tumor genome and the tumor grade, with a greater number of CNAs indicating a higher grade. Four CNAs were identified that could potentially be used as markers for aggressive MCTs in dogs (Table I) (30). Furthermore, the study screened for a KIT mutation often found in human MCTs and found that was often present in canine MCTs, and more so in tumors with a malignant phenotype (30). This is, therefore, potentially important for the development of diagnostic tests or optimization of prognostic schemes for human or canine patients with MCTs.

RT-qPCR technology has been employed to detect DEGs between MCT samples from two canine populations with cutaneous MCTs, one comprising dogs that died from MCT-associated causes and the other comprising dogs with surgically removed MCTs that survived to the end of the follow-up period (31) (Table II). A total of 375 DEGs were identified, a high number of which were associated with solute carrier transporters and UDP glucuronosyltransferases, both of which have been associated with chemotherapy-resistance in human oncology (31). 
Canine TVT. TVT is a cancer that is transmitted between dogs during coitus via the transplantation of allograft cancer cells from a tumor-bearing animal to the genitals of an unaffected animal (32). The disease affects the global canine population and has been in existence for thousands of years (32). Its longevity and transmissible nature have made it a popular cancer to study.

In 2016, a study used whole genome sequencing to examine the mitochondrial DNA (mtDNA) in tumor samples from dogs with TVT (32). The strength of this study lies in its large sample size; 449 TVT samples were used from a global population to gain a representative view of the distribution of the disease in all its genetic forms. The study demonstrated that there were five distinct genetic clusters, indicating that there were five different occasions in which horizontal mtDNA transfer occurred. This allowed the authors to map the spread of the disease throughout the world (32). Another whole genome study looked for the presence of single nucleotide variants and structural variants in the canine TVT genome by creating a large data library of inherited polymorphisms found in the genomes of wild and domestic canids throughout the world (33). Polymorphisms present in TVT but not common to genomes in the library are likely to be changes associated with the ability of TVT to evade detection in the body and instigate allograft creation (33). However, one issue with this method is that by creating a library of polymorphisms present in the canid genome, some founder-inherited alleles are read as somatic changes that have occurred since the origin of the TVT because these alleles are not present in the genome of the modern canid population. This misclassification overestimates the number of somatic variations present in TVT by $\sim 3.7 \%$ (33). Nonetheless, this study exemplifies how whole genome sequencing can be utilized to determine polymorphic changes over time in a large population. Although it lacks a human counterpart, study of this unique tumor type may offer special insights into certain aspects of tumor clonal evolution and tumor-host interaction.

Canine invasive urothelial carcinoma (iUC). Canine iUC is another excellent example of the use of comparative oncology in the improvement of the understanding and treatment of human cancers. Its success as a model for human iUC lies in its similar pathology, molecular biology and response to treatment (34). In addition, canine iUC has very similar sites of metastasis that occur with similar frequency to those in humans (34). Urothelial carcinoma in humans is defined as invasive if it has invaded at least as deep as the lamina propria (34). Much of the work on the comparative genomics of urothelial carcinoma has been based on the determination of genetic changes common to both canine and human forms with the aim of improving the diagnosis, understanding of the oncogenesis and refining its treatment.

In one study, oaCGH was used to target genetic changes consistent between human and canine forms of urothelial cell carcinoma (35). Whilst changes were found throughout the genome, the majority of changes comprised gains of CFA13 and CFA36, and losses of CFA19 of the canine genome. Numerous shared CNAs were identified between the human and canine genomes, and the frequencies of the aberrations were used to determine those most likely associated with urothelial carcinoma and those that represented conserved changes (35). The use of purebred dogs is beneficial due to their relative homogeneity, which makes it easier to differentiate relevant areas of the genome as there is less natural genetic variation. A gain of HSA 8 is the most common copy number change in human urothelial carcinoma and this is equivalent to a gain of CFA13, indicating there are similar genetic changes in human and canine forms of this cancer. The shared area of these two chromosomes is associated with the poly(A) binding protein cytoplasmic 1 gene. Other genes in shared areas of CNAs that merit further study include CDKN2A, S100 calcium-binding protein A8 (S100A8), S100A9 and LDL-receptor related protein $1 \mathrm{~B}$ (35). One possible limitation of this investigation was the method by which canine bladder cancers were sampled. Typically, samples were gained via cystoscopy, which takes a few layers of the bladder in which the tumor may invade; therefore, not all layers were available to be assessed, and some cases may have been at a later stage and more aggressive than diagnosed. This may have affected the correlation of the CNAs with the degree of severity reported by this study. More complete sampling of full thickness biopsy samples as is performed in human medicine may lead to more reliable results in this aspect.

A qNPA was developed to increase the yield and quality of genetic material harvested from formalin-fixed paraffin-embedded (FFPE) tissues, and was used to search for the increased expression of mRNA in canine iUC samples (36). The study found that in canine iUC, the EGFR and Erb-B2 receptor tyrosine kinase 2 genes are upregulated, as are the MET and estrogen receptor 1 genes, the latter two of which are known to be overexpressed in aggressive human bladder cancers (36). Therefore, the study concluded that using this assay method is effective for the retrieval of genetic material and measurement of gene expression in FFPE samples. However, another study that investigated the current methods used to extract DNA and RNA from FFPE samples concluded that significant issues remain with the extraction methods, leading to RNA and DNA samples being inadequate for ongoing processing (37). These issues include factors such as warm tissue ischemia and the duration between sample collection and fixation, which affect the quality of the genetic material (37). Hence, it appears that further study may be necessary to establish guidelines that will enable samples of the best possible quality to be obtained.

A gene expression microarray was employed to find expression patterns in the canine form of iUC that are known to be present in the human form (5). The study concluded that numerous genes were enriched in canine iUC and have similar expression patterns to those in the human form. Changes in the P53 pathway such as mutations and aberrations were also found to be common to both species (5).

\section{Review articles}

Five review papers were retrieved by the PubMed and CAB Abstracts searches and these reviews focus on different aspects of the literature on genomics and transcriptomics. The first paper chronologically examines the development of canine genetic studies, beginning with whole chromosomal 
studies using techniques such as FISH and moving on to more advanced technologies such as $\mathrm{CGH}$ that examine the genome down to the basepair level (38). By reviewing how these technologies have been used, the paper discusses how they have led to findings that have improved our understanding of canine evolution and oncology (38). Another review paper by the same group examines the functionality of different technologies and the type of genomic changes they are able to detect in greater detail (39). However, both reviews are now limited as they were published over a decade ago and, therefore, do not include the newer technologies and processes currently used. A third paper examines post-genomic techniques applied in canine oncology, systematically moving through different neoplastic types to review the technologies and science used to develop our current understanding of that cancer type (40). It highlights where technology has been successful and how technology may advance in the future to further understand these cancers. The fourth review paper is more targeted in that it focuses solely on transmissible cancers, namely Tasmanian devil facial tumor disease and TVT (41). The review focuses on how sequencing of the genomes of these tumors and mutation analysis have contributed to our knowledge of how these tumors originated and have developed over time. Finally, the fifth, most recent paper discusses the advantages that the presence of strong phenotypic homogeneity within canine breeds and extraordinary phenotypic variation between breeds offers to the identification of genes for a large number of canine maladies for which mouse models do not exist (3).

\section{Conclusions}

The aim of this review was to evaluate the current literature pertaining to veterinary genomics and transcriptomics in the area of oncology, including papers of value in human comparative oncology. From a database literature search using the terms 'veterinary', 'cancer' and 'genomics', 44 relevant articles were retrieved, the majority of which concerned canine tumors. These articles were reviewed to determine which cancer types were studied, the technology utilized, the genomic lesions targeted and the outcomes of the studies, and the principal findings were tabulated. In most studies, significant differences were found between the cancer genome studied and the control (usually non-cancer) genomes. To the best of our knowledge, this is the first review of this kind in the area of veterinary genomics and transcriptomics that focuses precisely on canine oncology. This review also concludes that genomic and transcriptomic technologies have been instrumental in developing our understanding of the origin, development and pathogenesis of a number of canine and human cancers, and has highlighted the areas in which more work needs to be done. Some studies were hampered by small sample sizes due to the difficulty in procuring enough samples or participants, while other issues lay in sample procurement, including issues with tumor sample collection, storage or the history accompanying the sample.

\section{Acknowledgements}

Not applicable.

\section{Funding}

No funding was received.

\section{Availability of data and materials}

Not applicable.

\section{Authors' contributions}

PL conceived and designed the review. BMH performed the literature review. PL and BMH confirm the authenticity of all the raw data. Both authors were involved in the preparation and revision of the manuscript. Both authors read and approved the final manuscript.

\section{Ethics approval and consent to participate}

Not applicable.

\section{Patient consent for publication}

Not applicable.

\section{Competing interests}

The authors declare that they have no competing interests.

\section{References}

1. Adams V, Evans K, Sampson J and Wood J: Methods and mortality results of a health survey of purebred dogs in the UK. J Small Anim Pract 51: 512-524, 2010

2. Pinho SS, Carvalho S, Cabral J, Reis CA and Gärtner F: Canine tumors: A spontaneous animal model of human carcinogenesis. Transl Res 159: 165-172, 2012

3. Ostrander EA, Dreger DL and Evans JM: Canine cancer genomics: Lessons for canine and human health. Annu Rev Anim Biosci 7: 449-472, 2019.

4. Davis BW and Ostrander EA: Domestic dogs and cancer research: A breed-based genomics approach. ILAR J 55: 59-68, 2014.

5. Katoh H, Shibata T, Kokubu A, Ojima H, Loukopoulos P, Kanai Y, Kosuge T, Fukayama M, Kondo T, Sakamoto M, et al: Genetic profile of hepatocellular carcinoma revealed by array-based comparative genomic hybridization: Identification of genetic indicators to predict patient outcome. J Hepatol 43: 863-874, 2005

6. Loukopoulos P, Shibata T, Katoh H, Kokubu A, Sakamoto M, Yamazaki K, Kosuge T, Kanai Y, Hosoda F, Imoto I, et al: Genome-wide array-based comparative genomic hybridization analysis of pancreatic adenocarcinoma: Identification of genetic indicators that predict patient outcome. Cancer Sci 98: 392-400, 2007.

7. O'Brien SJ: Pet project. Nature 438: 740, 2005.

8. Theisen A: Microarray-based comparative genomic hybridization (aCGH). Nature Educ 1: 45, 2008.

9. Dhawan D, Paoloni M, Shukradas S, Choudhury DR, Craig BA, Ramos-Vara JA, Hahn N, Bonney PL, Khanna C and Knapp DW: Comparative gene expression analyses identify luminal and basal subtypes of canine invasive urothelial carcinoma that mimic patterns in human invasive bladder cancer. PLoS One 10: e0136688, 2015.

10. Simpson S, Dunning MD, de Brot S, Grau-Roma L, Mongan NP and Rutland CS: Comparative review of human and canine osteosarcoma: Morphology, epidemiology, prognosis, treatment and genetics. Acta Vet Scand 59: 71, 2017.

11. Shao YW, Wood GA, Lu J, Tang QL, Liu J, Molyneux S, Chen Y, Fang H, Adissu H, McKee T, et al: Cross-species genomics identifies DLG2 as a tumor suppressor in osteosarcoma. Oncogene 38: 291-298, 2019. 
12. Phillips JC, Lembcke L and Chamberlin T: A novel locus for canine osteosarcoma (OSA1) maps to CFA34, the canine orthologue of human 3q26. Genomics 96: 220-227, 2010.

13. Sapierzyński R and Czopowicz M: The animal-dependent risk factors in canine osteosarcomas. Pol J Vet Sci 20: 293-298, 2017.

14. Loukopoulos P and Robinson WF: Clinicopathological relevance of tumour grading in canine osteosarcoma. J Comp Pathol 136: 65-73, 2007.

15. Loukopoulos P, Rozmanec M and Sutton RH: Cytological versus histopathological diagnosis in canine osteosarcoma. Vet Rec 157: 784, 2005.

16. Angstadt AY, Thayanithy V, Subramanian S, Modiano JF and Breen M: A genome-wide approach to comparative oncology: High-resolution oligonucleotide aCGH of canine and human osteosarcoma pinpoints shared microaberrations. Cancer Genet 205: 572-587, 2012.

17. Thomas R, Scott A, Langford CF, Fosmire SP, Jubala CM, Lorentzen TD, Hitte C, Karlsson EK, Kirkness E, Ostrander EA, et al: Construction of a $2-\mathrm{Mb}$ resolution BAC microarray for CGH analysis of canine tumors. Genome Res 15 : 1831-1837, 2005.

18. Phillips JC, Stephenson B, Hauck M and Dillberger J: Heritability and segregation analysis of osteosarcoma in the Scottish deerhound. Genomics 90: 354-363, 2007.

19. Meyer FR, Steinborn R, Grausgruber H, Wolfesberger B and Walter I: Expression of platelet-derived growth factor BB, erythropoietin and erythropoietin receptor in canine and feline osteosarcoma. Vet J 206: 67-74, 2015.

20. Wolfesberger B, Fuchs-Baumgartinger A, Hlavaty J, Meyer FR Hofer M, Steinborn R, Gebhard C and Walter I: Stem cell growth factor receptor in canine vs feline osteosarcomas. Oncol Lett 12: 2485-2492, 2016.

21. Dimopoulou M, Kirpensteijn J, Moens H and Kik M: Histologic prognosticators in feline osteosarcoma: A comparison with phenotypically similar canine osteosarcoma. Vet Surg 37: 466-471, 2008.

22. Fowles JS, Brown KC, Hess AM, Duval DL and Gustafson DL: Intra- and interspecies gene expression models for predicting drug response in canine osteosarcoma. BMC Bioinformatics 17: 93, 2016.

23. Thomas R, Seiser EL, Motsinger-Reif A, Borst L, Valli VE, Kelley K, Suter SE, Argyle D, Burgess K, Bell J, et al: Refining tumor-associated aneuploidy through 'genomic recoding' of recurrent DNA copy number aberrations in 150 canine non-Hodgkin lymphomas. Leuk Lymphoma 52: 1321-1335, 2011

24. Levsky JM and Singer RH: Fluorescence in situ hybridization: Past, present and future. J Cell Sci 116: 2833-2838, 2003.

25. Breen M and Modiano JF: Evolutionarily conserved cytogenetic changes in hematological malignancies of dogs and humans-man and his best friend share more than companionship. Chromosome Res 16: 145-154, 2008.

26. Suenaga M, Tomiyasu H, Watanabe M, Ogawa K, Motegi T, Goto-Koshino Y, Ohno K, Sugano S, Skorupski KA and Tsujimoto H: Comprehensive analysis of gene expression profiles reveals novel candidates of chemotherapy resistant factors in canine lymphoma. Vet J 228: 18-21, 2017.

27. Camus MS, Priest HL, Koehler JW, Driskell EA, Rakich PM, Ilha MR and Krimer PM: Cytologic criteria for mast cell tumor grading in dogs with evaluation of clinical outcome. Vet Pathol 53: 1117-1123, 2016.

28. Sledge DG, Webster J and Kiupel M: Canine cutaneous mast cell tumors: A combined clinical and pathologic approach to diagnosis, prognosis, and treatment selection. Vet J 215: 43-54, 2016.

29. Jark PC, Mundin DB, de Carvalho M, Ferioli RB, Anai LA, Marchi FA, Rogatto SR, Laufer-Amorim R and Tinucci-Costa M Genomic copy number variation associated with clinical outcome in canine cutaneous mast cell tumors. Res Vet Sci 111: 26-30, 2017.

30. Mochizuki H, Thomas R, Moroff S and Breen M: Genomic profiling of canine mast cell tumors identifies DNA copy number aberrations associated with KIT mutations and high histological grade. Chromosome Res 25: 129-143, 2017.

31. Giantin M, Baratto C, Marconato L, Vascellari M, Mutinelli F, Dacasto $\mathrm{M}$ and Granato A: Transcriptomic analysis identified up-regulation of a solute carrier transporter and UDP glucuronosyltransferases in dogs with aggressive cutaneous mast cell tumours. Vet J 212: 36-43, 2016.

32. Strakova A, Ní Leathlobhair M, Wang GD, Yin TT, Airikkala-Otter I, Allen JL, Allum KM, Bansse-Issa L, Bisson JL, Castillo Domracheva A, et al: Mitochondrial genetic diversity, selection and recombination in a canine transmissible cancer. Elife 5: e14552, 2016
33. Decker B, Davis BW, Rimbault M, Long AH, Karlins E, Jagannathan V, Reiman R, Parker HG, Drögemüller C, Corneveaux JJ, et al: Comparison against 186 canid wholegenome sequences reveals survival strategies of an ancient clonally transmissible canine tumor. Genome Res 25: 1646-1655, 2015.

34. Fulkerson CM, Dhawan D, Ratliff TL, Hahn NM and Knapp DW: Naturally occurring canine invasive urinary bladder cancer: A complementary animal model to improve the success rate in human clinical trials of new cancer drugs. Int J Genomics 2017: 6589529, 2017.

35. Shapiro SG, Raghunath S, Williams C, Motsinger-Reif AA, Cullen JM, Liu T, Albertson D, Ruvolo M, Bergstrom Lucas A, Jin J, et al: Canine urothelial carcinoma: Genomically aberrant and comparatively relevant. Chromosome Res 23: 311-331, 2015.

36. Davis B, Schwartz M, Duchemin D, Barrett JC and Post G: Validation of a multiplexed gene signature assay for diagnosis of canine cancers from formalin-fixed paraffin-embedded tissues. J Vet Intern Med 31: 854-863, 2017.

37. Granato A, Giantin M, Ariani P, Carminato A, Baratto C, Zorzan E, Vascellari M, Bozzato E, Dacasto M and Mutinelli F: DNA and RNA isolation from canine oncologic formalin-fixed, paraffin-embedded tissues for downstream '-omic' analyses: Possible or not? J Vet Diagn Invest 26: 117-124, 2014.

38. Breen M: Canine cytogenetics-from band to basepair. Cytogen Genome Res 120: 50-60, 2008.

39. Breen M: Update on genomics in veterinary oncology. Topics Companion Anim Med 24: 113-121, 2009.

40. Ceciliani F, Roccabianca P, Giudice C and Lecchi C: Application of post-genomic techniques in dog cancer research. Mol Biosyst 12: 2665-2679, 2016.

41. Jones EA, Cheng Y and Belov K: The origin, dynamics, and molecular evolution of transmissible cancers. Adv Genom Genet 5: 317-326, 2015.

42. Ferraresso S, Aricò A, Sanavia T, Da Ros S, Milan M, Cascione L, Comazzi S, Martini V, Giantin M, Di Camillo B, et al: DNA methylation profiling reveals common signatures of tumorigenesis and defines epigenetic prognostic subtypes of canine diffuse large B-cell lymphoma. Sci Rep 7: 11591, 2017.

43. Courtay-Cahen C, Platt SR, De Risio L and Starkey MP. Preliminary analysis of genomic abnormalities in canine meningiomas. Vet Comp Oncol 6: 182-192, 2008

44. Dickinson PJ, York D, Higgins RJ, LeCouteur RA, Joshi N and Bannasch D: Chromosomal aberrations in canine gliomas define candidate genes and common pathways in DOGS AND HUmans. J Neuropathol Exp Neurol 75: 700-710, 2016.

45. Thomas R, Duke SE, Bloom SK, Breen TE, Young AC, Feiste E, Seiser EL, Tsai PC, Langford CF, Ellis P, et al: A cytogenetically characterized, genome-anchored $10-\mathrm{Mb} \mathrm{BAC}$ set and CGH array for the domestic dog. J Hered 98: 474-484, 2007.

46. Wang G, Wu M, Maloneyhuss MA, Wojcik J, Durham AC, Mason NJ and Roth DB: Actionable mutations in canine hemangiosarcoma. PLoS One 12: e0188667, 2017.

47. Laufer-Amorim R, Fonseca-Alves CE, Villacis RAR, Linde SAD, Carvalho M, Larsen SJ, Marchi FA and Rogatto SR: Comprehensive genomic profiling of androgen-receptor-negative canine prostate cancer. Int J Mol Sci 20: 1555, 2019.

48. Zapata I, Moraes LE, Fiala EM, Zaldivar-Lopez S, Couto CG, Rowell JL and Alvarez CE: Risk-modeling of dog osteosarcoma genome scans shows individuals with Mendelian-level polygenic risk are common. BMC Genomics 20: 226, 2019.

49. Gardner HL, Sivaprakasam K, Briones N, Zismann V, Perdigones N, Drenner K, Facista S, Richholt R, Liang W, Aldrich $\mathrm{J}$, et al: Canine osteosarcoma genome sequencing identifies recurrent mutations in DMD and the histone methyltransferase gene SETD2. Commun Biol 2: 266, 2019.

50. Maués T, El-Jaick KB, Costa FB, Freitas PVS, Moreira AS, Castro L, Ferreira MLG and Ferreira AMR: Could polymorphisms in ABCB1 gene represent a genetic risk factor for the development of mammary tumors in dogs? Vet J 248: 58-63, 2019.

51. Korpershoek E, Dieduksman DAER, Grinwis GCM, Day MJ, Reusch CE, Hilbe M, Fracassi F, Krol NMG, Uitterlinden AG, deKlein A, etal: Molecular alterations in dog pheochromocytomas and paragangliomas. Cancers (Basel) 11: 607, 2019.

52. Kosack L, Wingelhofer B, Popa A, Orlova A, Agerer B, Vilagos B, Majek P, Parapatics K, Lercher A, Ringler A, et al: The ERBB-STAT3 axis drives tasmanian devil facial tumor disease. Cancer Cell 14: 125-139.e9, 2019. 
53. Singer-Berk MH, Knickelbein KE, Lounsberry ZT, Crausaz M, Vig S, Joshi N, Britton M, Settles ML, Reilly CM, Bentley E, et al: Additional evidence for DDB2 T338M as a genetic risk factor for ocular squamous cell carcinoma in horses. Int J Genomics 2019: 3610965, 2019.

54. Megquier K, Turner-Maier J, Swofford R, Kim JH, Sarver AL, Wang C, Sakthikumar S, Johnson J, Koltookian M, Lewellen M, et al: Comparative genomics reveals shared mutational landscape in canine hemangiosarcoma and human angiosarcoma. Mol Cancer Res 17: 2410-2421, 2019.

55. Amin SB, Anderson KJ, Boudreau CE, Martinez-Ledesma E, Kocakavuk E, Johnson KC, Barthel FP, Varn FS, Kassab C, Ling X, et al: Comparative molecular life history of spontaneous canine and human gliomas. Cancer Cell 37: 243-257.e7, 2020.

56. Grimes JA, Prasad N, Levy S, Cattley R, Lindley S, Boothe HW, Henderson RA and Smith BF: A comparison of microRNA expression profiles from splenic hemangiosarcoma, splenic nodular hyperplasia, and normal spleens of dogs. BMC Vet Res 12: 272, 2016.

57. Mahoney JA, Fisher JC, Snyder SA and Hauck ML: Feasibility of using gene expression analysis to study canine soft tissue sarcomas. Mamm Genome 21: 577-582, 2010.
58. Lee KH, Park HM, Son KH, Shin TJ and Cho JY: Transcriptome signatures of canine mammary gland tumors and its comparison to human breast cancers. Cancers (Basel) 10: 317, 2018.

59. Rahman M, Lai Y, Husna AA, Chen HW, Tanaka Y, Kawaguchi H, Hatai H, Miyoshi N, Nakagawa T, Fukushima R and Miura N: Transcriptome analysis of dog oral melanoma and its oncogenic analogy with human melanoma. Oncol Rep 43: 16-30, 2020.

60. Panchal K, Koringa P, Sabara P, Bhatia D, Jakhesara S and Joshi C: Expression profiling revealed keratins and interleukins as potential biomarkers in squamous cell carcinoma of horn in Indian bullocks (Bos indicus). 3 Biotech 10: 92, 2020.

61. Sabara PH, Jakhesara SJ, Panchal KJ, Joshi CG and Koringa PG: Transcriptomic analysis to affirm the regulatory role of long non-coding RNA in horn cancer of Indian zebu cattle breed Kankrej (Bos indicus). Funct Integr Genomics 20: 75-87, 2020.

This work is licensed under a Creative Commons Attribution-NonCommercial-NoDerivatives 4.0 International (CC BY-NC-ND 4.0) License. 